\title{
TOPOGRAPHIC DISTRIBUTION OF LYMPHOCYTE INFILTRATE IN TRIPLE NEGATIVE TUMORS AND ITS IMPACT ON THE SURVIVAL OF THE PATIENT WITH BREAST CANCER
}

Jose Peixoto1, Marcos Duarte Guimarães², Maria Aparecida Seabra³, Olávio Campos Júnior³, Ana Carolina Ferraz Pascoal ${ }^{1}$

${ }^{1}$ Hospital do Câncer de Pernambuco - Recife (PE), Brazil.

${ }^{2}$ A.C. Camargo Cancer Center - São Paulo (SP), Brazil.

${ }^{3}$ Laboratório de Imunopatologia Keiso Asami - São Paulo (SP), Brazil.

Introduction: Tumor infiltrating lymphocytes (TIL) are generally measured using subjective methods unable to differentiate subpopulations and to locate immune cells in the tumor microenvironment. The identification and location of these cells is of great importance as they present different responses to immune stimuli. Objective: to analyze the presence of TIL in early-stage breast cancer of the triple negative molecular subtype, and to evaluate the association of TIL with the overall survival time (OS). Methods: a cross-sectional study was carried out at the Hospital de Câncer de Pernambuco (HCP), where patients diagnosed with triple negative breast cancer were selected between 2009 and 2013. TIL evaluation was performed by two methods: on slides stained with hematoxylin and eosin (H\&E), by two pathologists blindly and independently, and on slides submitted to immunohistochemistry, with CD3 and CD8 lymphocyte marking. In this case, the sample was then subjected to computerized histophotometry and morphometric analysis. Results: 87 patients were included, of which 22 patients had an event in the follow-up period. The evaluation of TIL, by two pathologists, showed regular agreement between the evaluators, with an intraclass correlation coefficient of 0.574 and $\mathrm{p}=0.001$. CD3+ and CD8+ lymphocytes were in greater quantity in the intra tumor area compared to those outside the tumor margin (extra tumor). When the association between the presence of lymphocytes and the patient's OS was analyzed, a directly proportional relationship with this survival was observed, that is, the greater the amount of lymphocytes, the lower the risk of death. Conclusion: results suggest that there is a correlation between the tumor lymphocyte infiltrate of triple negative breast cancer and OS. As this tumor subtype has a poor prognosis and does not have specific target therapy, TIL analysis can be explored as a prognostic marker for the treatment of breast cancer. 